\title{
The association between oxidative stress and endothelial dysfunction in early childhood patients with Kawasaki disease
}

\author{
Takamichi Ishikawa* (D) and Keigo Seki
}

\begin{abstract}
Background: Oxidative stress has recently been shown to play an important role in the development of arteriosclerosis in patients with Kawasaki disease (KD); however, no study has investigated this association in early childhood patients with KD. In this study, we evaluated prospectively the association between the levels of oxidative stress and the endothelial function in early childhood patients with KD.

Methods: We compared the derivatives of reactive oxygen metabolites (ROM), flow-mediated dilatation (FMD), and biological characteristics in a population of 50 children: 10 patients with $\mathrm{KD}$ and coronary artery lesions (CAL) (group 1), $15 \mathrm{KD}$ patients without CAL (group 2), and 25 healthy age- and sex-matched children (group 3).

Results: The median age of all KD children at study enrollment was 6.8 (IQR 4.4-8.2) years. ROM levels were significantly higher in group $1(p<0.001)$ and group $2(p=0.004)$ than in group 3 . The \%FMD of group $1(p<0.001)$ and group $2(p=0.026)$ was significantly lower than that of group 3. There was a significant negative correlation between ROM and \%FMD ( $r=-0.60, p<0.001)$. A multiple linear regression analysis identified In-ROM (standardized coefficient $=-0.403, p=0.043$ ) and total fever duration (standardized coefficient $=-0.413, p=0.038$ ) as significant determinants of \%FMD in the patients with KD.

Conclusions: Our study suggests that oxidative stress is strongly associated with endothelial dysfunction in early childhood patients with KD. Furthermore, we found that the longer the fever duration, the higher the risk of oxidative stress-induced endothelial dysfunction in these children.
\end{abstract}

Keywords: Oxidative stress, Kawasaki disease, Endothelial dysfunction, Total fever duration, Children

\section{Background}

Kawasaki disease (KD) is characterized by systemic vasculitis, and occurs most frequently in infants and young children. A history of KD has recently been indicated as a possible risk factor for the early onset of arteriosclerosis. Endothelial dysfunction is one of the earliest manifestations of arteriosclerosis [1,2] and has been demonstrated in KD children with or without coronary artery lesions (CAL) after acute illness [3, 4]. While these mounting evidences suggest that there is premature arteriosclerosis in patients with a history of $\mathrm{KD}$, the underlying mechanisms are still undefined.

\footnotetext{
* Correspondence: ishikawa@hama-med.ac.jp

Department of Pediatrics, Hamamatsu University School of Medicine, 1-20-1 Handayama, Higashi-ku, Hamamatsu 431-3192, Japan
}

Oxidative stress is caused by the presence of reactive oxygen species (ROS). Excessive ROS production represents endothelial and smooth muscle dysfunction, which leads to the progression of atherosclerosis $[5,6]$. Recently, the derivatives of reactive oxygen metabolites (ROM) test, which mainly consists of measuring hydroperoxide levels as a marker of ROS, has been used to directly assay the total oxidant capacity [7-9].

It has been increasingly recognized that oxidative stress plays an integral role in the development of arterial endothelial dysfunction and the risk of premature arteriosclerosis in KD patients. However, most of the previously reported studies investigated the association between oxidative stress and the endothelial function in adolescents and adults with KD late after the onset of the disease [10-12]. In the present study, we evaluated 
the association between the levels of oxidative stress and the endothelial function in early childhood patients with KD. In addition, we determined whether any differences existed in the factors associated with oxidative stress and the endothelial function in these children.

\section{Methods}

We enrolled two groups of KD patients with and without CAL and a group of age- and sex-matched healthy controls (1:1 matching). All of the patients with KD were diagnosed in or referred to the Hamamatsu University Hospital in Japan. The KD patients who met the following criteria were recruited: (1) a diagnosis of KD that was established by the Japanese Kawasaki Disease Research Committee, and (2) an interval from the initial onset of illness of up to five years. We obtained the data for all subjects from their clinical records. As a control group, we recruited healthy children who had undergone a health checkup with no evidence of disease history and with a normal physical examination. Controls matched according to sex and date of birth (within 1 year) were selected from these children. The subjects were recruited during the study period between April 2015 and September 2016. The institutional Ethics Committee approved the study and the parents of all of the participants provided their written informed consent.

We classified the participants into three groups: group 1 comprised the patients with CAL, whether persistent or regressed, group 2 comprised the patients without CAL at any time, and group 3 comprised age- and sexmatched healthy control children. The coronary artery segments measured by echocardiography included the internal lumen diameters of the left main coronary artery, left anterior descending artery, and right coronary artery. The coronary artery dimensions were normalized for body surface area as $\mathrm{z}$-scores and calculated by regression eqs. [13]. Patients were considered to have CAL if any coronary artery segment had a $\mathrm{z}$-score $\geq 2.5$ and were excluded to have CAL if any coronary artery segment had a z-score $<2.5$.

Fasting total cholesterol (TC), high-density lipoprotein cholesterol (HDL-C), low-density lipoprotein cholesterol (LDL-C), and triglyceride levels were determined. The plasma TC, HDL-C, triglyceride, and uric acid levels were determined by conventional enzymatic methods. The concentration of LDL-C was calculated by the Friedewald formula. The glycosylated hemoglobin A1c was measured by electrophoresis. The plasma Nterminal-pro-B-type natriuretic peptide (NT-proBNP) concentration was measured on an Elecsys 2010 analyzer with a chemiluminescent immunoassay kit (Roche Diagnostics GmbH, Mannheim, Germany). We also calculated the TC/HDL-C ratio by dividing TC by HDL-C. The $\mathrm{C}$-reactive protein (CRP) level was measured with a highly sensitive assay, which is a particle-enhanced immunoturbidimetric assay consisting of an anti-CRP monoclonal antibody coupled to latex microparticles (Roche Diagnostics). The assay is standardized against a CRM 470 reference preparation for proteins in human serum and has a functional sensitivity of $0.1 \mathrm{mg} / \mathrm{L}$.

The principle of the ROM test has been described previously $[7,8]$. We measured hydroperoxide levels as serum ROM levels in children in the present study using a Free Radical Elective Evaluator (FREE ${ }^{\circ}$; Wismerll Co. Ltd., Tokyo, Japan). The ROM test spectrophotometrically detects the oxidization of N,N-diethyl-para-phenylenediamine as a chromogenic substrate by radicals converted from hydroperoxide. To measure ROM, a 20$\mu \mathrm{L}$ serum sample and $1 \mathrm{~mL}$ of buffered solution (R2 kit reagent) were gently mixed in a cuvette, and $20 \mu \mathrm{L}$ of chromogenic substrate (R1 kit reagent) was then added to the cuvette. After mixing well, the cuvette was immediately incubated in the thermostatic block of the analyzer for $5 \mathrm{~min}$ at $37{ }^{\circ} \mathrm{C}$ and the absorbance at $505 \mathrm{~nm}$ was recorded [14]. Measurements are expressed as an arbitrary unit called the Carratelli unit (U.CARR) such that 1 U.CARR corresponds to $0.8 \mathrm{mg} / \mathrm{L}_{2} \mathrm{O}_{2}$. The normal reference level of ROM was 250 to 300 U.CARR $[7,8]$.

Endothelial function was measured using flowmediated dilatation (FMD) performed by a single trained researcher (T.I.) blinded to the subject's status by a randomization code, according to the guidelines and standards $[15,16]$. The diameter of the brachial artery was measured on high-resolution, two-dimensional ultrasound images obtained by Philips HD11XE (Philips Medical Systems, Andover, MA, USA) devices with a $12 \mathrm{~L}$ linear array transducer. A sphygmomanometer cuff used for vascular occlusion was positioned distal to the ultrasound probe and inflated to a pressure of $200 \mathrm{mmHg}$ for $5 \mathrm{~min}$, then deflated suddenly to induce an increased blood flow. Brachial artery diameter was measured from the anterior to the posterior interface between the media and adventitia. The mean diameter of the brachial artery was calculated from three cardiac cycles gated with an electrocardiogram. The peak percent FMD (\%FMD) was defined as the percentage change relative to that of the initial resting scan.

A 10-min rest was provided after the FMD scan for vessel recovery, and the non-endothelial-dependent brachial artery dilatation was assessed with twodimensional imaging before and 3 to $4 \mathrm{~min}$ after the administration of sublingual glyceryl trinitrate (GTN) spray $(0.3 \mathrm{mg})$. The peak \%GTN was defined as the percentage change in the arterial diameter after the administration of GTN relative to the baseline vessel diameter.

The same examiner performed the measurements of the intima-media thickness (IMT), FMD, and GTN 
throughout the study. The IMT of the common carotid artery was measured at high resolution with B-mode ultrasound images obtained by the same devices and transducer. For the measurements of IMT, longitudinal images of the carotid arteries were obtained [17]. The distance between the leading edges of the lumen-intima interface and the media-adventitia interface of the B-mode frame was considered. A software program (Qlab; Philips Medical Systems) was used to analyze the IMT distance automatically at 64 points within a $10-\mathrm{mm}$ segment. The value given was the calculated arithmetic mean IMT.

Data are presented as the mean \pm standard deviation and median (interquartile range: IQR) as appropriate. Data normality was initially verified using the ShapiroWilk test allowing the use of parametric statistical analysis. To compare the differences between the groups, a one-way analysis of variance (ANOVA) and post hoc Tukey's test were used for the parametric variables, the Kruskal-Wallis test with post hoc comparison by Dunn's multiple comparison test for the non-parametric variables, and Fisher's exact test for the categorical variables. When a significant difference was indicated by ANOVA, the specific source of the difference was identified using either the Mann-Whitney $U$ test or paired t-test, with Bonferroni correction. For comparison of non-parametric variables between two groups, the Mann-Whitney $U$ test was used. The correlations were analyzed using Spearman's correlation coefficient by rank. Because the ROM levels were not normally distributed, we calculated the natural logarithmic transformed ROM as $\ln -\mathrm{ROM}$ to be used for regression analysis. A multiple regression analysis was performed in $\mathrm{KD}$ patients to examine the associations between \%FMD and the clinical parameters, including the number of days with a fever before diagnosis, total fever duration, white blood cell (WBC) count, CRP level, age, sex, body mass index (BMI), and ln-ROM after a univariate analysis. For this purpose, a linear regression model was used in a stepwise method with a probability of 0.05 to enter and 0.10 to remove. Significance was defined as a $p$ value of less than 0.05 .

\section{Results}

A total of 50 children were studied. Group 1 comprised 10 patients, six of whom had persistent coronary aneurysms and four of whom had regressed aneurysms. None had symptoms of myocardial ischemia and none required coronary artery interventions. Group 2 comprised 15 patients with KD and excluded CAL. All of the 25 patients with KD had received intravenous immunoglobulin (IVIG) during the acute phase of illness, and six were continuing to take long-term, oral low-dose aspirin. Of the $25 \mathrm{KD}$ patients, 18 children completely responded to a single IVIG therapeutic session, and seven patients required a second dose of IVIG because of a persistent fever; however, of these seven patients, six failed to respond to the second IVIG therapy session. In these six children, one patient received plasma exchange therapy, four patients received prednisolone therapy and one patient required infliximab therapy. We performed coronary angiography (CAG) in all ten patients in Group 1, not in the remaining 15 children without CAL. The median age of all KD children (groups 1 and 2) at study enrollment was 6.8 years (IQR 4.4-8.2 years). The patients with KD were studied at a median time interval of 3.9 years (IQR 1.4-4.8 years) from the onset of disease. Group 3 comprised 25 age- and sex-matched healthy children.

The baseline and biological characteristics of all patients are shown in Table 1. There was no significant difference in any of the parameters among the three groups. The levels of TC, HDL-C, LDL-C, TC/HDL-C ratio, triglyceride, uric acid, hemoglobin A1c, and NTproBNP were all within normal limits and were not significantly different among the three groups.

The ROM levels differed significantly among the three groups, being significantly higher in group 1 (median 394 U.CARR, IQR 383-458 U.CARR) than in group 3 (median 298 U.CARR, IQR 268-327 U.CARR, $p<0.001$ ). The ROM levels in group 2 (median 353 U.CARR, IQR 328-412 U.CARR) were also significantly greater than in group $3(p=0.004)$ (Fig. 1).

The \%FMD was significantly lower in group $1(6.0 \pm$ $1.5 \%)$ than in group $3(12.9 \pm 4.7 \%, \mathrm{p}<0.001)$. The \%FMD was also reduced significantly in group $2(9.2 \pm 2.9 \%)$ in comparison to group $3(p=0.026)$. There were no significant differences in \%GTN $(p=0.611)$ and IMT $(p=0.425)$ between the three groups (Table 2). The intraobserver variability in the measurements of the FMD, GTN and IMT was $6.8 \%, 5.4 \%$, and $4.6 \%$, respectively. Importantly, there was a significant negative correlation between ROM and \%FMD ( $\mathrm{r}=-0.60, p<0.001)$ (Fig. 2).

In the patients with $\mathrm{KD}$, the number of days with a fever before diagnosis was greater and the total fever duration was significantly longer in group 1 than in group $2(p=0.003$ and $p<0.001$, respectively). The serum CRP concentration in group 1 was greater than that in group $2(p=0.001)$. However, there was no significant difference in the WBC count between the two groups $(p=0.268)$ (Table 3$)$. We also investigated the correlations between \%FMD and these parameters added to age, sex, BMI, and $\ln -\mathrm{ROM}$ in patients with $\mathrm{KD}$. Using a univariate analysis, we found that the number of days with a fever before diagnosis, total fever duration, CRP levels, and ln-ROM inversely correlated with \%FMD. To establish the independent predictors of FMD in patients with $K D$, a stepwise multiple linear regression analysis including these variables was performed; lnROM (standardized coefficient $=-0.403, \quad p=0.043$ ) and total fever duration (standardized coefficient =- 
Table 1 Clinical and biological characteristics of the study group

\begin{tabular}{|c|c|c|c|c|}
\hline & Group $1(n=10)$ & Group $2(n=15)$ & Group $3(n=25)$ & $p$ Value \\
\hline Age (years) & $7.5^{*}(4.3-11.7)$ & $6.7^{*}(5.0-7.6)$ & $6.4^{*}(5.8-9.4)$ & 0.611 \\
\hline Sex (male/female) & $4 / 6$ & $8 / 7$ & $12 / 13$ & 0.832 \\
\hline Age at diagnosis of KD (years) & $2.9^{*}(1.2-6.4)$ & $2.1^{*}(1.0-3.2)$ & NA & 0.952 \\
\hline Follow-up interval (years) & $3.4^{*}(1.1-3.9)$ & $4.5^{*}(4.0-4.9)$ & NA & 0.243 \\
\hline Height (cm) & $122.0 \pm 29.2$ & $111.8 \pm 15.5$ & $119.7 \pm 15.7$ & 0.450 \\
\hline Weight (kg) & $28.4 \pm 13.6$ & $21.0 \pm 6.3$ & $23.0 \pm 7.5$ & 0.514 \\
\hline BMI (kg/m2) & $17.2 \pm 2.3$ & $16.5 \pm 1.6$ & $15.7 \pm 1.5$ & 0.120 \\
\hline Systolic BP (mm Hg) & $94.4 \pm 5.7$ & $95.8 \pm 6.0$ & $96.4 \pm 7.0$ & 0.807 \\
\hline Diastolic BP (mm Hg) & $54.0 \pm 5.4$ & $53.6 \pm 6.9$ & $55.7 \pm 5.2$ & 0.234 \\
\hline HR (beats/min) & $87.0 \pm 17.4$ & $91.9 \pm 17.8$ & $86.5 \pm 13.5$ & 0.324 \\
\hline $\mathrm{TC}(\mathrm{mg} / \mathrm{dL})$ & $183.6 \pm 30.6$ & $168.9 \pm 21.6$ & $170.6 \pm 22.7$ & 0.247 \\
\hline $\mathrm{HDL}-\mathrm{C}(\mathrm{mg} / \mathrm{dL})$ & $61.0 \pm 26.0$ & $58.8 \pm 11.5$ & $62.8 \pm 12.2$ & 0.335 \\
\hline LDL-C (mg/dL) & $97.9 \pm 13.1$ & $92.3 \pm 15.5$ & $90.6 \pm 24.3$ & 0.593 \\
\hline $\mathrm{TC} / \mathrm{HDL}-\mathrm{C}$ & $3.3 \pm 0.8$ & $3.0 \pm 0.6$ & $2.8 \pm 0.6$ & 0.458 \\
\hline Triglyceride (mg/dL) & $94.3 \pm 47.1$ & $88.3 \pm 37.7$ & $79.4 \pm 39.5$ & 0.483 \\
\hline Uric Acid (mg/dL) & $4.5 \pm 0.9$ & $3.9 \pm 0.8$ & $3.9 \pm 1.2$ & 0.180 \\
\hline Hemoglobin A1c (\%) & $5.2 \pm 0.4$ & $5.4 \pm 0.3$ & $5.4 \pm 0.3$ & 0.869 \\
\hline NT-pro BNP (pg/mL) & $42.8 \pm 19.7$ & $61.2 \pm 24.8$ & $55.5 \pm 21.8$ & 0.192 \\
\hline
\end{tabular}

*The values are presented as the median (interquartile range)

0.413, $p=0.038)$ were independently associated with \%FMD (Table 4).

\section{Discussion}

The present study showed the following: (1) a significant increase in levels of ROM, an oxidative stress marker, in early childhood patients after KD; (2) a

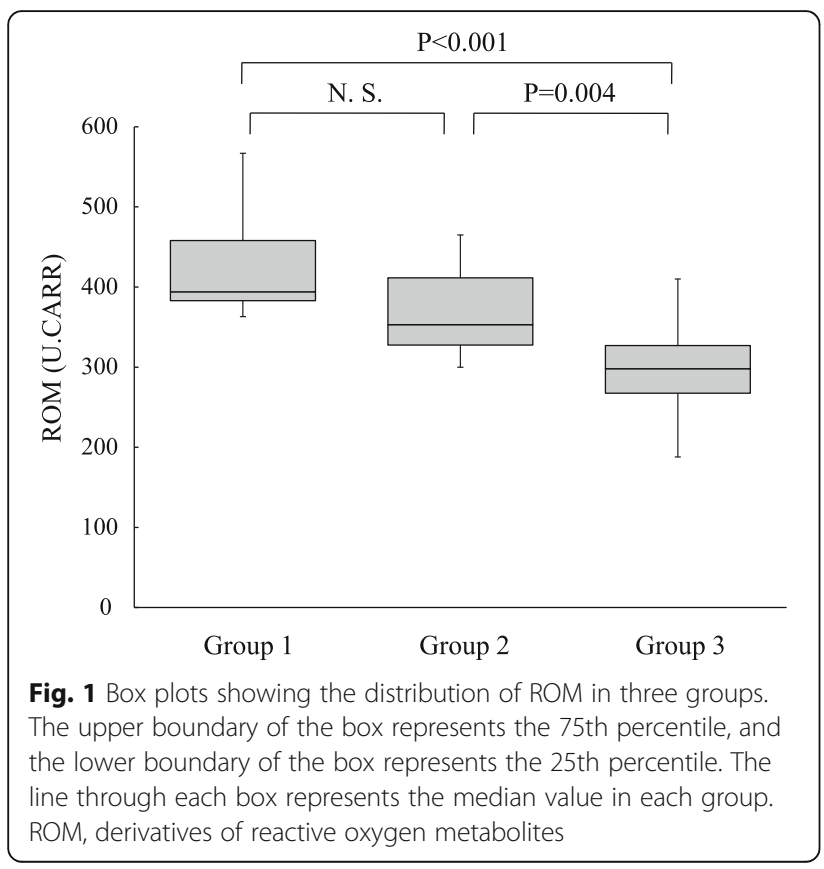

significant negative correlation between ROM and \%FMD in the study population; and (3) that ROM and total fever duration were closely associated with \%FMD in the KD children.

Our study population comprised younger patients whose median age and elapsed time from the initial onset were 6.8 and 3.9 years, respectively. By contrast, the study populations in most previously reported studies that assessed oxidative stress and endothelial function comprised teenage or adult patients [10-12]. This is the first report to investigate the association between oxidative stress and the endothelial function in early childhood patients with KD.

The ROM levels in the acute stage of KD have recently been described by Yahata et al. [18]. Although ROM levels were reduced by IVIG therapy in these patients, the levels at two weeks after the IVIG treatment (430 U.CARR) remained higher than the normal range. However, the state of oxidative stress between the acute and late phase of $\mathrm{KD}$ was unclear. The results of the present study confirmed that the ROM levels in KD patients with and without CAL were significantly greater than in normal controls, and suggest that oxidative stress is persistent after the acute phase of $\mathrm{KD}$.

We demonstrated in this early childhood study that there was a significant negative correlation between ROM and \%FMD, which has been widely regarded as a marker of the endothelial function. It is well known that the grade 
Table 2 Comparison of FMD, GTN-mediated dilatation, and Carotid IMT in the three groups

\begin{tabular}{|c|c|c|c|c|c|c|c|}
\hline & \multirow{2}{*}{$\begin{array}{l}\text { Group } 1 \\
(n=10)\end{array}$} & \multirow{2}{*}{$\begin{array}{l}\text { Group } 2 \\
(n=15)\end{array}$} & \multirow{2}{*}{$\begin{array}{l}\text { Group } 3 \\
(n=25)\end{array}$} & \multirow{2}{*}{$\begin{array}{l}\text { p Value } \\
\text { (ANOVA) }\end{array}$} & \multicolumn{3}{|c|}{$p$ Value (post hoc test) } \\
\hline & & & & & 1 vs. 3 & 2 vs. 3 & 1 vs. 2 \\
\hline FMD (\%) & $6.0 \pm 1.5$ & $9.2 \pm 2.9$ & $12.9 \pm 4.7$ & $<0.001$ & $<0.001$ & 0.026 & 0.082 \\
\hline GTN-mediated dilatation (\%) & $22.4 \pm 6.2$ & $25.0 \pm 5.0$ & $25.3 \pm 5.0$ & 0.611 & & & \\
\hline Carotid IMT (mm) & $0.44 \pm 0.03$ & $0.44 \pm 0.03$ & $0.42 \pm 0.04$ & 0.425 & & & \\
\hline
\end{tabular}

of the endothelial function is a predictor of cardiovascular outcomes in adults. Higashi et al. demonstrated the role of oxidative stress in the endothelial dysfunction. They described one of the possible mechanisms whereby reduced nitric oxide (NO) and increased oxidative stress impaired the endothelium-dependent vasodilation [19]. Accumulating evidence suggests that endothelial dysfunction may be caused by the accelerated inactivation of nitric oxide by ROS, which reduces the bioavailability of NO [20]. Our findings of a correlation between ROM and \%FMD strongly support these speculations. Based on our results, ROM, which reflects oxidative stress, may be a useful biomarker to evaluate the presence of endothelial dysfunction in children with $\mathrm{KD}$.

Endothelial dysfunction is an early feature of both arteriosclerosis and vascular diseases in humans $[1,2]$. In addition, endothelial dysfunction is an earlier manifestation of arteriosclerosis than carotid IMT thickening. In the present study, there was no significant difference in the IMT between the KD patients and the controls. Carotid IMT thickening is postulated to be secondary to the changes in the arterial walls after diffuse vasculitis due to KD [21]. It is conceivable that
IMT thickening was not detected in this study because our study population was younger than that in previous studies [22, 23]. In young children, especially those within five years of the onset of KD, FMD impairment occurs prior to intima-media complex thickening. Our results suggest that oxidative stress may cause functional changes in the arterial wall in the premature arteriosclerosis earlier than the anatomical changes in KD patients within five years after the onset of illness.

In this study, among KD patients with CAL, the inflammatory process was more severe than that observed in the remaining children (greater number of days with a fever before diagnosis and the total fever duration and increased CRP levels). Dalla et al. also reported that the patients with coronary arterial involvement had increased CRP levels at the time of presentation of the illness [24]. The American Heart Association and American Academy of Pediatrics guidelines recommend that IVIG be administered to children with KD within the first 10 days of illness, and if possible, within the first seven days of illness [21]. Coronary artery aneurysms occur significantly more often in patients with a delayed

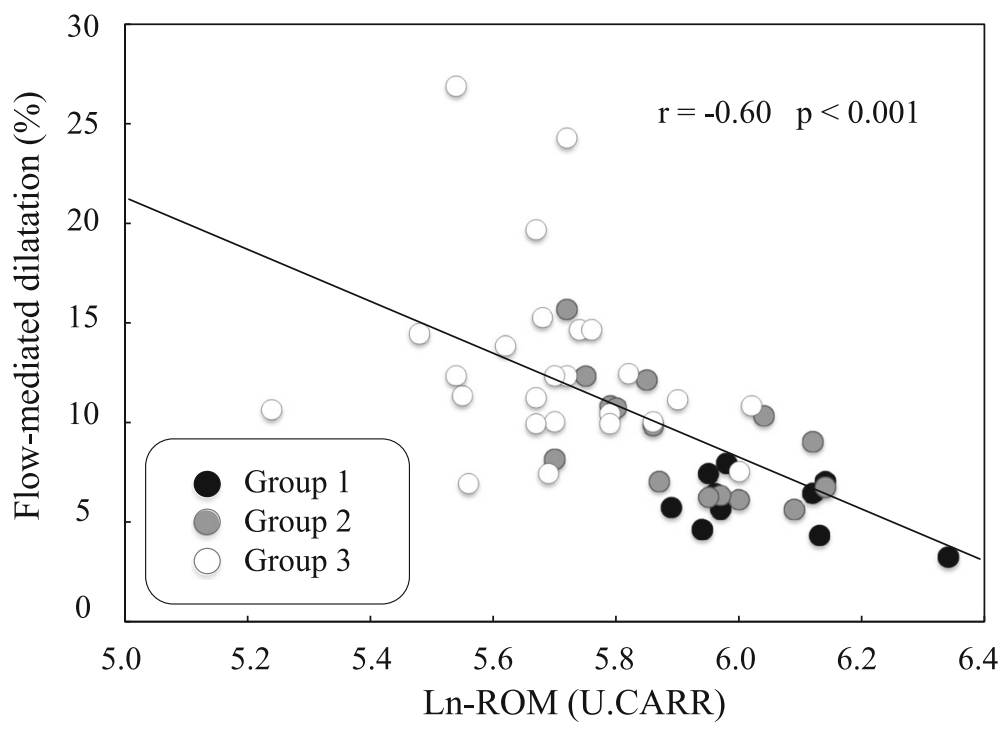

Fig. 2 A scatter plot and correlation between \%FMD and ROM. FMD, flow-mediated dilatation; ROM, derivatives of reactive oxygen metabolites 
Table 3 Clinical characteristics of patients with and without coronary involvement

\begin{tabular}{llll}
\hline & Group $1(n=10)$ & Group 2 $(n=15)$ & $p$ Value \\
\hline Days of fever before diagnosis & $6.4 \pm 1.8$ & $4.6 \pm 0.9$ & 0.003 \\
Total fever duration (days) & $10.5 \pm 2.5$ & $5.5 \pm 1.6$ & $<0.001$ \\
WBC (cells/ $\mathrm{LL})$ & $16300^{*}(12525-19,800)$ & $14600^{*}(12250-16,150)$ & 0.268 \\
CRP $(\mathrm{mg} / \mathrm{dL})$ & $14.6^{*}(10.9-19.2)$ & $7.6^{*}(5.2-8.1)$ & 0.001 \\
\hline
\end{tabular}

*The values are presented as the median (interquartile range)

diagnosis of $\mathrm{KD}$, especially when the total duration of fever is longer than eight days $[25,26]$. Importantly, the current study documented that ROM and total fever duration were independently associated with \%FMD in the patients with KD. These results suggest that a prolonged inflammatory process causes not only coronary artery aneurysm but also systemic endothelial dysfunction caused by persistent oxidative stress. The association between increased oxidative stress and subclinical inflammation amplifies the signals that lead to vascular damage $[27,28]$. Proinflammatory cytokines and advanced glycation end-products generate the intracellular ROS responsible for the activation of redox-sensitive transcription factors and phenotypical changes in endothelial cells, including the expression of adhesion molecules and tissue factor and the release of proinflammatory cytokines that characterize endothelial dysfunction [29]. Our study showed that a longer duration of fever is associated a higher risk of oxidative stress-induced endothelial dysfunction. We believe that the duration of fever is as important with CAL as without CAL in regard to endothelial dysfunction and the genesis of arteriosclerosis. For this reason, the patients with prolonged fever during $\mathrm{KD}$ should take care regarding arteriosclerosis, even if they have no CAL. Therefore, shortening the total fever duration is important for the prevention of early arteriosclerosis in the KD patients.

This study has some limitations. First, it was a small study of only 25 children with KD and 25 controls, so the findings should be considered as preliminary. Larger studies are needed to confirm the differences in oxidative stress and the endothelial function between control children and the patients with KD. Second, the fact that patients were not receiving the same treatment may affect the results of the present study. In particular, additional treatment such as steroid and infliximab and plasma exchange may have influenced the results. Third, we performed CAG to only Group 1 children. We may have been able to demonstrate coronary arteriosclerosis or atherosclerosis in more detail if all patients with KD had received CAG. Fourth, we did not assess the levels of oxidative stress and the endothelial function in the different phases of KD. Further investigations with a long follow-up period are needed to clarify the true risk for arteriosclerosis in patients with $\mathrm{KD}$.

\section{Conclusions}

The present study evaluated the oxidative stress and the endothelial function after KD in the youngest patients reported to date. In conclusion, our study suggests that oxidative stress may have a pathogenic role in the functional changes of the arterial wall even in early childhood, especially within five years of the onset of KD. In addition, we found that a longer duration of fever results in a higher risk of endothelial dysfunction caused by persistent oxidative stress. Patients with a persistent or recurrent fever during $\mathrm{KD}$, such as non-responders to IVIG, may require long-term follow-up to monitor the genesis of arteriosclerosis.

Table 4 Univariate analysis and multiple regression analysis of the relation between \%FMD and variables in patients with KD

\begin{tabular}{|c|c|c|c|c|}
\hline & \multicolumn{2}{|l|}{ Univariate analysis } & \multicolumn{2}{|l|}{ Multiple regression analysis } \\
\hline & Regression coefficient & $p$ Value & Standardized coefficient (95\% Cl) & p Value \\
\hline Age (year) & 0.027 & 0.449 & & \\
\hline Sex & 0.261 & 0.103 & & \\
\hline $\mathrm{BMI}\left(\mathrm{kg} / \mathrm{m}^{2}\right)$ & -0.173 & 0.204 & & \\
\hline Days of fever before diagnosis & -0.489 & 0.007 & & \\
\hline Total fever duration (days) & -0.671 & $<0.001$ & $-0.413(-0.732$ to -0.022$)$ & 0.038 \\
\hline 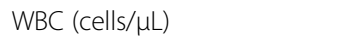 & -0.167 & 0.213 & & \\
\hline CRP $(\mathrm{mg} / \mathrm{dL})$ & -0.517 & 0.004 & & \\
\hline Ln-ROM (U. CARR) & -0.667 & $<0.001$ & $-0.403(-14.741$ to -0.274$)$ & 0.043 \\
\hline
\end{tabular}




\section{Abbreviations}

ANOVA: Analysis of variance; BMI: Body mass index; CAL: Coronary artery lesions; CRP: C-reactive protein; ECG: Electrocardiogram; FMD: Flow-mediated dilatation; GTN: Glyceryl trinitrate; HDL-C: High-density lipoprotein cholesterol; IMT: Intima-media thickness; IQR: Interquartile range; IVIG: Intravenous immunoglobulin; KD: Kawasaki disease; LDL-C: Low-density lipoprotein cholesterol; NO: Nitric oxide; NT-proBNP: N-terminal-pro-B-type natriuretic peptide; ROM: Reactive oxygen metabolites; ROS: Reactive oxygen species; SD: Standard deviation; TC: Fasting total cholesterol;

U.CARR: Carratelli unit; WBC: White blood cell

\section{Acknowledgements}

We thank Edanz Group (www.edanzediting.com/ac) for editing a draft of this manuscript.

\section{Funding}

This work was supported by Grant-in-Aid for Scientific Research (KAKENH 15 K09646) from the Ministry of Education, Science, and Culture of Japan and the Japan Society for the Promotion of Science. The funding sources had no involvement in the conduct of the research or preparation of the article.

\section{Availability of data and materials}

Raw data supporting the obtained results are available from the corresponding author.

\section{Authors' contributions}

Tl: designed, drafted, and wrote the manuscript, performed the statistical analysis, and collected the data. KS: made substantial contributions to conception and design and collected the data. Both authors read and approved the final manuscript.

\section{Ethics approval and consent to participate}

The ethics committee of the Hamamatsu University School of Medicine approved the study. Written informed consent was obtained from the parents of all of the participants.

\section{Consent for publication}

Not applicable.

\section{Competing interests}

The authors declare that they have no competing interests.

\section{Publisher's Note}

Springer Nature remains neutral with regard to jurisdictional claims in published maps and institutional affiliations.

\section{Received: 27 June 2017 Accepted: 31 January 2018}

\section{Published online: 09 February 2018}

\section{References}

1. Bürrig KF. The endothelium of advanced arteriosclerotic plaques in humans. Arterioscler Thromb. 1991:11:1678-89.

2. Ross R. Atherosclerosis: an inflammatory disease. N Engl J Med. 1999:340:115-26.

3. Niboshi A, Hamaoka K, Sakata K, Yamaguchi N. Endothelial dysfunction in adult patients with a history of Kawasaki disease. Eur J Pediatr. 2008;167:189-96

4. AlHuzaimi A, Al Mashham Y, Potts JE, De Souza AM, Sandor GG. EchoDoppler assessment of arterial stiffness in pediatric patients with Kawasaki disease. J Am Soc Echocardiogr. 2013;26:1084-9.

5. Griendling KK, FitzGerald GA. Oxidative stress and cardiovascular injury: part I: basic mechanisms and in vivo monitoring of ROS. Circulation. 2003;108:1912-6.

6. Griendling KK, FitzGerald GA. Oxidative stress and cardiovascular injury: part II: animal and human studies. Circulation. 2003:108:2034-40.

7. Cesarone MR, Belcaro G, Carratelli M, Cornelli U, De Sanctis MT, Incandela L, Barsotti A, Terranova R, Nicolaides AA. Simple test to monitor oxidative stress. Int Angiol. 1999;18:127-30.
8. lamele L, Fiocchi R, Vernocchi A. Evaluation of automated spectrophotometric assay for reactive oxygen metabolites in serum. Clin Chem Lab Med. 2002;40:673-6.

9. Ridker PM, Brown NJ, Vaughan DE, Harrison DG, Mehta JL. Established and emerging plasma biomarkers in the prediction of first atherothrombotic events. Circulation. 2004;109:IV6-19.

10. Cheung YF, K O, Woo CW, Armstrong S, Siow YL, Chow PC, Oxidative CEW. Stress in children late after Kawasaki disease: relationship with carotid atherosclerosis and stiffness. BMC Pediatr. 2008;8:1-8.

11. Hamaoka A, Hamaoka K, Yahata T, Fujii M, Ozawa S, Toiyama K, Nishida M, Itoi T. Effects of HMG-CoA reductase inhibitors on continuous postinflammatory vascular remodeling late after Kawasaki disease. J Cardiol. 2010:56:245-53.

12. Tobayama H, Takahashi K, Fukunaga H, Matsui K, Tanaka N, Harada M, Furukawa T, Oda H, Akimoto K, Kishiro M, Shimizu T. Analysis of arterial function in adults with a history of Kawasaki disease. J Cardiol. 2013;61:330-5.

13. McCrindle BW, Li JS, Minich LL, Colan SD, Atz AM, Takahashi M, Vetter VL, Gersony WM, Mitchell PD, Newburger JW, Investigators PHN. Coronary artery involvement in children with Kawasaki disease: risk factors from analysis of serial normalized measurements. Circulation. 2007;116:174-9.

14. Kujiraoka T, Satoh Y, Ayaori M, Shiraishi Y, Arai-Nakaya Y, Hakuno D, Yada H, Kuwada N, Endo S, Isoda K, Adachi T. Hepatic extracellular signal-regulated kinase 2 suppresses endoplasmic reticulum stress and protects from oxidative stress and endothelial dysfunction. J Am Heart Assoc. 2013;2:e000361.

15. Corretti MC, Anderson TJ, Benjamin EJ, Celermajer D, Charbonneau F, Creager MA, Deanfield J, Drexler H, Gerhard-Herman M, Herrington D, Vallance P, Vita J, Vogel R. International Brachal artery reactivity task force. Guidelines for the ultrasound assessment of endothelial-dependent flowmediated vasodilation of the brachial artery: a report of the international brachial artery reactivity task force. J Am Coll Cardiol. 2002;39:257-65.

16. Kobayashi K, Akishita M, Yu W, Hashimoto M, Ohni M, Toba K. Interrelationship between non-invasive measurements of atherosclerosis: flow-mediated dilation of brachial artery, carotid intima-media thickness and pulse wave velocity. Atherosclerosis. 2004;173:13-8.

17. Hashimoto M, Eto M, Akishita M, Kozaki K, Ako J, lijima K, Kim S, Toba K, Yoshizumi M, Ouchi Y. Correlation between flow-mediated vasodilatation of the brachial artery and intima-media thickness in the carotid artery in men. Arterioscler Thromb Vasc Biol. 1999;19:2795-800.

18. Yahata T, Suzuki C, Hamaoka A, Fujii M, Hamaoka K. Dynamics of reactive oxygen metabolites and biological antioxidant potential in the acute stage of Kawasaki disease. Circ J. 2011;75:2453-9.

19. Higashi Y, Noma K, Yoshizumi M, Kihara Y. Endothelial function and oxidative stress in cardiovascular disease. Circ J. 2009;73:411-8.

20. Cai $H$, Harrison DG. Endothelial dysfunction in cardiovascular diseases: the role of oxidant stress. Circ Res. 2000;87:840-4.

21. Newburger JW, Takahashi M, Gerber MA, Gewitz MH, Tani LY, Burns JC, Shulman ST, Bolger AF, Ferrieri P, Baltimore RS, Wilson WR, Baddour LM, Levison ME, Pallasch TJ, Falace DA, Taubert KA. Committee on rheumatic fever, endocarditis and Kawasaki disease; council on cardiovascular disease in the young; American Heart Association; American Academy of Pediatrics. Diagnosis, treatment, and long-term management of Kawasaki disease: a statement for health professionals from the committee on rheumatic fever, endocarditis and Kawasaki disease, council on cardiovascular disease in the young, American Heart Association. Circulation. 2004;110:2747-71.

22. Noto N, Okada T, Yamasuge M, Taniguchi K, Karasawa K, Ayusawa M, Sumitomo N, Harada K. Noninvasive assessment of the early progression of atherosclerosis in adolescents with Kawasaki disease and coronary artery lesions. Pediatrics. 2001;107:1095-9.

23. Cheung YF, Ho MH, Tam SC, Yung TC. Increased high sensitivity C reactive protein concentrations and increased arterial stiffness in children with a histry of Kawasaki disease. Heart. 2004:90:1281-5.

24. Dalla Pozza R, Bechtold S, Urschel S, Kozlik-Feldmann R, Netz H. Subclinical atherosclerosis, but normal autonomic function after Kawasaki disease. J Pediatr. 2007;151:239-43.

25. Rowley AH, Gonzalez-Crussi F, Gidding SS, Duffy CE, Shulman ST. Incomplete Kawasaki disease with coronary artery involvement. J Pediatr. 1987:110:409-13.

26. Kim T, Choi W, Woo CW, Choi B, Lee J, Lee K, Son C, Lee J. Predictive risk factors for coronary artery abnormalities in Kawasaki disease. Eur J Pediatr. 2007;166:421-5. 
27. Davi G, Guagnano MT, Ciabattoni G, Basili S, Faco A, Marinopiccoli M, Nutini $M$, Sensi S, Patrono C. Platelet activation in obese women: role of inflammation and oxidant stress. JAMA. 2002;288:2008-14.

28. Steinberg D. Atherogenesis in perspective: hypercholesterolemia and inflammation as partners in crime. Nat Med. 2002;8:1211-7.

29. Brunner H, Cockcroft JR, Deanfield J, Donald A, Ferrannini E, Halcox J, Kiowski W, Luscher TF, Mancia G, Natali A, Oliver JJ, Pessina AC, Rizzoni D, Rossi GP, Salvetti A, Spieker LE, Taddei S, Webb DJ. Endothelial function and dysfunction. Part Il: association with cardiovascular risk factors and diseases. A statement by the working group on Endothelins and endothelial factors of the European Society of Hypertension. J Hypertens. 2005;23:233-46.

Submit your next manuscript to BioMed Central and we will help you at every step:

- We accept pre-submission inquiries

- Our selector tool helps you to find the most relevant journal

- We provide round the clock customer support

- Convenient online submission

- Thorough peer review

- Inclusion in PubMed and all major indexing services

- Maximum visibility for your research

Submit your manuscript at www.biomedcentral.com/submit
Biomed Central 